\title{
Ripples in relativity
}

\section{Traveling at the Speed of Thought: \\ Einstein and the Quest for Gravitational \\ Waves \\ by Daniel Kennefick \\ Princeton University Press: 2007. 334 pp. $\$ 35, £ 22.95$}

\section{Clifford Will}

Gravitational physicists are eagerly awaiting the moment when gravitational-wave astronomy becomes a reality. More than half-a-billion US dollars have been sunk into ground-based, laser interferometric gravitational-wave observatories, and NASA and the European Space Agency are contemplating spending even more on the

Laser Interferometer Space Antenna. Quite bold, when you consider that so far we have only indirect experimental evidence for gravitational waves. And, as Daniel Kennefick reminds us in his entertaining book, Traveling at the Speed of Thought, it was not so long ago that relativity theorists debated whether gravitational waves exist at all.

According to Einstein's general theory of relativity, the waves are ripples in the warpage or curvature of space-time that can be emitted by suitably moving masses; they travel with the same speed as light and, when they encounter a pair of masses, they cause them to move relative to each other, albeit by minuscule amounts.

Einstein in 1916 was the first to calculate the gravitational waves emitted by a system such as a rotating dumbbell. He showed that there are wave modes that can travel with arbitrary speed, which inspired Arthur Eddington in 1922 to coin the "speed of thought" phrase that forms the title of Kennefick's book.

We now understand these modes to be oscillations of the coordinates used to label the points in space-time, something that is allowed by the general covariance of Einstein's general theory of relativity but that has absolutely no physical consequences. Only waves of spacetime curvature are physical. In hindsight, it seemed to take an inordinately long time almost 40 years - to sort this out. Kennefick does a good job of describing the issues, the players and the many steps needed to get this and other issues squared away for good.

In 1936, Einstein thought he had proved the non-existence of gravitational waves. In what is now a legendary episode, he and his assistant Nathan Rosen at Princeton University, New Jersey, submitted the proof to the Physical Review, but Einstein was so appalled that the editor had dared to send it to an anonymous referee that he withdrew the paper and never published in that journal again.

But in Kennefick's telling, the story gets even more interesting. The referee's report, plus the results of a discussion between noted cosmologist H. P. Robertson and physicist Leopold Infeld, another of Einstein's assistants, may have convinced Einstein that the 'proof' was actually wrong. When he and Rosen later pub-

bell, the stars in a binary system are in free fall, and some relativists held that there would be no wave emission from such systems. Even among those who were convinced that there would be waves, there was debate about how to calculate their effects quantitatively. This is usually called the 'quadrupole formula controversy', named after the formula that gives the leading order effects. The debate over the validity of this formula raged rather strongly from the 1950 s on, until the 1978 announcement by Joseph Taylor that the measured decay of the orbit of the 'binary pulsar' agreed with the quadrupole formula (today it agrees to $0.3 \%$ ).

It is often said that history is written by the winners, and as a participant myself in the quadrupole controversies - on the winning side - I confess to having occasionally poohpoohed the role of the sceptics. The historian's role is to provide a richer perspective, and to elucidate the full dynamics of the story, taking advantage of hindsight, but also armed with interviews and a balanced reading of the documentary evidence. I must say that I learned much from Kennefick's retelling of the quadrupole wars and now appreciate better the viewpoints of the sceptics and their important contributions to the final resolution.

Kennefick, who is both a relativist and a science historian, writes in an engaging manner, although the book would be rather tough going for a lay reader. There is a fair amount of technical talk, plus some jargon from history and philosophy of science. Still, I recommend this book to readers with more than a passing interest in physics and its history. One thing I would have liked is a comparison of the controver-

lished their paper in the Journal of the Franklin Institute, it now claimed exactly the opposite - that the waves did exist. In a relativistic version of Watergate, people have wondered who was the 'deep throat' of Physical Review. Although suspicion naturally pointed towards Robertson, there was no clear proof. Kennefick helped find the smoking gun by discovering in Robertson's papers at the California Institute of Technology in Pasadena a carbon copy of the report, and by talking Physical Review into finding the old log books where then-editor John Tate had recorded sending the EinsteinRosen paper to Robertson. For the experts, Kennefick reproduces Robertson's referee's report in an appendix.

Kennefick also recounts another debate over whether gravitational waves would be emitted by binary star systems. Unlike a rotating dumb- sies described in this book with other modern scientific controversies, and not just with controversies of the era of Galileo or Newton. Are the difficulties described here unique to general relativity, or are they typical whenever one is exploring uncharted scientific territory?

Today, theorists are calculating waves many orders of approximation beyond the simple quadrupole formula, to determine the precise signal emitted from the decaying orbits of black-hole binaries (one of the leading candidates for detection) possibly within a decade. We feel we are now on solid theoretical ground, so it is not a stretch to call this 'applied' general relativity. Kennefick's book reminds us of the complex path that brought us to this point. Clifford Will is a professor of physics at Washington University, St Louis, Missouri 63130-4899, USA. 